\title{
Common haemostasis issues in major bleeding and critical illness
}

\author{
Authors: Divyansh Gulati, ${ }^{A}$ Alex Novak ${ }^{B}$ and Simon J Stanworth ${ }^{C}$
}

\begin{abstract}
Haemostatic abnormalities are common in the critically ill or bleeding patient, including haemorrhage-related coagulopathies, disseminated intravascular coagulation and thrombocytopenia, among other pathologies. In this article we aim to outline some of the causes of these clotting abnormalities, highlighting recent advancements in knowledge and new insights into their clinical management, with the aim of optimising diagnostic and therapeutic strategies.
\end{abstract}

\section{Introduction}

Normal haemostasis reflects a balance between the naturally occurring procoagulant and anticoagulant mechanisms found in the body. Any shift in this balance or interference in the control mechanisms may result in either an increased risk of bleeding or a thrombotic tendency. This article attempts to summarise some key issues of haemostasis as applied to common practice in critically ill patients. To provide a framework for this discussion, we have provided two fictitious case scenarios that are not infrequently faced by clinicians. One covers major bleeding (see Box 1) and the other critical illness (see Box 2). The management challenges in these patients are different. The patient with life-threatening major bleeding may develop a range of abnormalities in clotting but the immediate focus of care in these patients is to maintain plasma volume and minimise further deterioration, in coagulation.

\section{Major haemorrhage}

Recent years have seen the widespread adoption of major haemorrhage protocols to guide shop-floor clinicians when treating severe bleeding, based around the use of packed red blood cells (PRBCs) and fresh frozen plasma (FFP) delivered in fixed ratios, which aim to treat the coagulopathy that frequently accompanies major bleeding and limit its initial development. An important recent study, the Pragmatic Randomized Optimal

Authors: ${ }^{A}$ registrar, emergency medicine and ambulatory care, Oxford University Hospitals NHS Foundation Trust, Oxford, UK; ${ }^{B}$ consultant in emergency medicine and ambulatory care, Oxford University Hospitals NHS Foundation Trust, Oxford, UK; ${ }^{C}$ consultant haematologist, Department of Haematology, Oxford University Hospitals NHS Foundation Trust, Oxford, UK
Platelet and Plasma Ratios (PROPPR) trial, is often referred to as establishing the benefits of empirical transfusion of PRBCs and FFP in fixed 1:1 ratio in the treatment of patients with major haemorrhage due to trauma, and an approach adopted within the current British Society of Haematology (BSH) guidelines, the interpretation of the results in this study continues to be debated for several reasons. ${ }^{1,2}$ In addition to evaluating a fixed high ratio of plasma to red cells, the intervention arm also included platelets. The trial primary outcome reported no difference in overall survival between early administration of plasma, platelets and red blood cells in a 1:1:1 ratio compared to 1:1:2, although findings were reported as significant for other secondary outcomes including death from exsanguination. ${ }^{2}$

Another area of considerable research focus has centred on coagulation abnormalities in trauma-related major bleeding. Typically these have been defined by abnormalities of the prothrombin time (PT) and reported to occur in some patients before fluid and transfusion therapy has commenced and

\section{Key points}

Major haemorrhage due to trauma can be associated with a coagulopathy in part based on the early depletion of fibrinogen and fibrinolysis driven by activation of Protein C

Standard coagulation tests may be of limited use in major haemorrhage as they are often insensitive in detecting coagulopathy

Viscoelastic hemostatic assays (VHAs) may potentially provide a more global assessment of coagulation status with faster turnaround of results but their utility is not fully established

Disseminated intravascular coagulation (DIC) can be challenging to diagnose using standard laboratory tests and requires clinical judgment

Thrombocytopenia is common in critical care patients thresholds for platelet replacement depend on the underlying clinical picture

KEYWORDS: Major haemorrhage, coagulopathy, disseminated intravascular coagulation 
Box 1. Case report 1

A 43-year-old man was transferred to a major trauma centre having sustained crush injuries to his pelvis following an accident on a building site. On arrival he was hypotensive and tachycardic, with a lactate of 5.3 and a normal haemoglobin obtained on a venous blood gas. Laboratory blood tests were sent and he was treated with intravenous tranexamic acid and transfused with 2 units each of packed red cells (PRBCS) and fresh frozen plasma (FFP). Computed tomography identified a splenic rupture and pelvic fractures with large amount of blood in his abdominal cavity. He was then urgently transferred to theatre for stabilisation of his pelvic fractures and management of his splenic injury.

therefore is not dilutional. This research into trauma-induced coagulopathy (TIC), and in particular the acute traumatic coagulopathy (ATC) identified by Brohi et al, has suggested early depletion of fibrinogen and fibrinolysis driven by activation of Protein $\mathrm{C}$ may be significant driving mechanisms. ${ }^{3,4}$ The important role of hyperfibrinolysis provides a rationale for early use of tranexamic acid, as documented clearly in the CRASH-2 trial. ${ }^{5}$ Other research is ongoing to evaluate the therapeutic use of concentrated forms of fibrinogen such as cryoprecipitate and fibrinogen concentrate. ${ }^{6}$

It is important to recognise that these studies have been undertaken in trauma and may not apply in other clinical settings of major bleeding. The coagulation abnormalities in patients with bleeding due to variceal bleeding in patients with cirrhosis is poorly studied and in these patients acute changes in vascular pressures due to 'aggressive' fluid therapy are unlikely to be beneficial. Haemostatic changes in pregnancy mean that the management of postpartum haemorrhage (PPH) should also be considered differently from trauma., ${ }^{7,8}$ There is a shift towards a prothrombotic state, with increases in the circulating concentrations of all clotting factors (except Factor XI), and especially fibrinogen, von Willebrand factor (VWF) and Factor VIII, which are reflected in laboratory assays and whole blood viscoelastic hemostatic assays (VHA) measurements. ${ }^{9,10,11}$

\section{Diagnostic limitations in haemostasis}

The BSH guidelines suggest that after the initial transfusions of FFP (and red cells), further transfusion management should be guided by results of conventional laboratory-based tests (PT, APTT, fibrinogen).

There are many general limitations when documenting abnormalities of PT and APTT tests and the PT and APTT provide only a very limited assessment of haemostatic status. Firstly, they measure the time taken for a clot to form, which occurs when only $5 \%$ of total thrombin generation has occurred. Secondly, they reflect only the procoagulant factors and are unaffected by the levels of natural anticoagulants. Thirdly, they are insensitive to modest but potentially clinically relevant levels of other factors, although the most frequently used test, the PT, is sensitive to functionally unimportant reductions in factor VII. Finally, they are poor at identifying a prothrombotic state. ${ }^{12}$

The $\mathrm{BSH}$ guidelines recommend clotting tests being taken every 30-60 minutes during a major haemorrhage episode, although this can be difficult to manage in clinical practice, and the time taken to receive the results of standard
Box 2. Case report 2

A 19-year-old woman was admitted to hospital with a 1-day history of fever and malaise, followed by a rapid deterioration and collapse. Clinical examination revealed a purpuric rash over lower limbs and trunk. She was treated empirically with intravenous antibiotics and fluids, and admitted to the intensive care unit. Blood cultures subsequently confirmed meningococcal septicaemia. During admission her condition deteriorated, with a concomitant drop in platelets and a prolonged PT on her clotting profile. This was suspected as being due to disseminated intravascular coagulation and she was treated with an unfractionated heparin infusion.

lab tests (often over $45 \mathrm{~min}$ ) also limits their utility in emergency situations. ${ }^{1}$ The potential value of conventional laboratorybased testing in individual patients is not as a single isolated measure of coagulation but for serial monitoring of a dynamic process. $^{13,14}$

Near patient tests (eg viscoelastic haemostatic assays [VHAs] such as TEG/ROTEM) are often considered promising alternative diagnostic tests but their exact role remains to be defined in clinical trials. ${ }^{1}$ VHAs may potentially provide a more global assessment of coagulation status with faster turnaround of results (approximately $10 \mathrm{~min}$ ); however, the main evidence for the value of VHAs has been in cardiac surgery. In trauma, the ITACTIC randomised trial is ongoing; this multinational European study aims to assess their use in the management of traumatic haemorrhage. ${ }^{15}$

\section{Critical illness}

\section{Disseminated intravascular coagulation}

A number of disorders are frequently encountered in the critical care environment that can result in disseminated intravascular coagulation (DIC). DIC remains a poorly understood and recognised entity. ${ }^{19}$ International Society for Thrombosis and Haemostasis (ISTH) guidelines exist to help standardised diagnosis of DIC, but their value in clinical practice remains less clear. Precipitating factors include sepsis, malignancies, trauma, obstetric complications and autoimmune disorders, with sepsis being by the far the most common. ${ }^{16}$ DIC remains a challenge to identify clinically, as there are no clinical findings sufficiently sensitive or specific to accurately diagnose the condition, and its overall course can vary considerably with different manifestations of the disease. ISTH has proposed a scoring method for overt and non-overt DIC based on standard laboratory assays (see Table 1). ${ }^{17}$ While these still remain the main method of establishing a diagnosis of DIC, they are often not sensitive enough to detect its presence $-50 \%$ of cases of DIC have a normal PT for example. Low or rapidly decreasing platelet levels are the most common abnormal haematological feature, though these too may be normal in some cases. ${ }^{18}$

Initial treatment of DIC is essentially based on supportive care and treating the underlying cause (eg initiating antibiotic administration and source control in sepsis-related DIC). In early cases this may be sufficient to halt the process and restore normal haemostasis; however, in more severe cases haemostatic derangements may persist. ${ }^{19}$ 
Table 1. ISTH scoring system for overt disseminated intravascular coagulation

\begin{tabular}{l} 
Platelet count \\
$>100,000 / \mu \mathrm{L}$ ( 0 points) \\
$50,000 / \mu \mathrm{L}-100,000 / \mu \mathrm{L}$ ( 1 point) \\
$<50,000 / \mu \mathrm{L}$ ( 2 points) \\
Increase in fibrin markers \\
No change (0 points) \\
Moderate rise ( 2 points) \\
Strong rise ( 3 points) \\
Prothrombin time prolongation \\
$\leq 3$ seconds (0 points) \\
$>3$ seconds but <6 seconds ( 1 point) \\
$>6$ seconds ( 2 points) \\
Fibrinogen Level \\
Greater than $1 \mathrm{~g} / \mathrm{L}$ ( 0 points) \\
Less than $1 \mathrm{~g} / \mathrm{L}$ ( 1 point) \\
\hline 0-4 points: DIC is not overt, repeat score in $1-2$ days \\
ISTH = International Society for Thrombosis and Haemostasis
\end{tabular}

\section{Thrombocytopenia in critical care}

A number of conditions commonly encountered in critical care can result in a drop in the circulating platelet count, including sepsis, DIC, thrombotic microangiopathy, and heparin-induced thrombocytopenia (HIT) - the incidence of thrombocytopenia in intensive care units (ICUs) has been shown to range from $13-50 \% .{ }^{20}$ Typical thresholds for replacement therapy are at $<30-50 \times 10^{9} / \mathrm{L}$ in a patient suspected of bleeding, or $<10$ whether bleeding suspected or not. In patients with platelet dysfunction, eg due to antiplatelet medication, uraemia, or severe liver failure, the picture may be more complex, as platelet levels may not correspond to activity/effectiveness. However, the recent PATCH trial reported evidence of harm associated with use of platelet transfusions in the setting of intracranial bleeding in patients on antiplatelet drugs who were treated with platelet infusions. ${ }^{21}$ The reasons for this outcome are unclear, but serve to emphasise the point that platelet transfusions are biological products with a range of potential immunomodulatory and proinflammatory consequences.

\section{Prolonged coagulation times in non-bleeding patients}

Prolonged coagulation times are common in the ICU, with an international normalised ratio (INR) $\geq 1.5$ (INR - a ratio to standardise the PT test that allows for differences between laboratories so that the test results can be compared) detected in almost two-thirds of patients; however, providing clear evidencebased recommendations for prophylactic use of plasma in non-bleeding critically ill patients remains challenging. ${ }^{22} \mathrm{FFP}$ is widely used in the treatment of coagulopathy in the bleeding patient, but it is likely that much of this is inappropriate, and it is estimated that over one-third of its use in current UK ICU practice is outside standard recommendation. ${ }^{11}$ Recently, $\mathrm{BSH}$ published guidelines on the use of FFP and cryoprecipitate in absence of bleeding. ${ }^{23}$ The Intensive Care Study of Coagulopathy-1 (ISOC1) study described significant prophylactic FFP use in 1,923 critical care admissions with coagulopathies defined by standard coagulation assays. ${ }^{24}$ The follow-up ISOC-2 study evaluated the use of an alternative global assay termed 'thrombin generation' (TG), that might provide more information on the summation of procoagulant activities or better address the net balance between procoagulant and anticoagulant forces. ${ }^{25}$ Plasma samples were collected and analysed from 306 patients admitted to intensive care with prolonged PT. Overall, despite prolonged PT, 47.8\% patients had endogenous thrombin potential (ETP) within normal limits. Another recent randomised trial by De Pietri et al evaluated the use of TEG in 60 patients with cirrhosis and significant coagulopathy who were undergoing an invasive procedure. The authors reported that TEG-guided transfusion strategy led to a significantly lower use of blood products without an increase in bleeding complications. ${ }^{26}$ These publications support the need for better tests to determine whether interventions can be withheld safely in groups of nonbleeding patients with prolonged PTR to provide a rationale that would avoid complications of unnecessary transfusions of plasma to non-bleeding patients.

\section{Summary}

Challenges remain in the identification and management of haemostasis in major haemorrhage and critical illness. Future developments should include the use of alternative diagnostic tests, and increased understanding of the role and efficacy of triggered therapeutic interventions may greatly enhance our ability to manage these conditions.

\section{Funding}

The research was supported by the National Institute for Health Research (NIHR) Oxford Biomedical Research Centre (BRC).

\section{References}

1 Hunt BJ, Allard S, Keeling D et al. A practical guideline for the haematological management of major haemorrhage. $\mathrm{Br}$ ] Haematol 2015;170:788-803.

2 Holcomb JB, Tilley BC, Baraniuk S et al. Transfusion of plasma, platelets, and red blood cells in a 1:1:1 vs a 1:1:2 ratio and mortality in patients with severe trauma: the PROPPR randomized clinical trial. JAMA 2015;313:471-82.

3 Brohi K, Singh J, Heron M, Coats T. Acute traumatic coagulopathy. J Trauma 2003;54:1127-30.

4 Davenport RA, Guerreiro M, Frith D et al. Activated protein C drives the hyperfibrinolysis of acute traumatic coagulopathy. Anesthesiology 2017:126:115-27.

5 CRASH-2 trial collaborators, Shakur H, Roberts I et al. Effects of tranexamic acid on death, vascular occlusive events, and blood transfusion in trauma patients with significant haemorrhage (CRASH-2): a randomised, placebo-controlled trial. Lancet 2010:376:23-32.

6 Novak A, Stanworth SJ, Curry N. Do we still need cryoprecipitate? Cryoprecipitate and fibrinogen concentrate as treatments for major hemorrhage - how do they compare? Expert Rev Hematol 2018;11:351-60.

7 World Health Organization. Maternal mortality. WHO, 2016.

8 Mavrides E, Allard S, Chandraharan E et al on behalf of the Royal College of Obstetricians and Gynaecologists. Prevention and management of postpartum haemorrhage. BJOG 2016;124:e106-e149. 
9 O'Riordan MN, Higgins JR. Haemostasis in normal and abnormal pregnancy. Best Pract Res Clin Obstet Gynaecol 2003;17:385-96.

10 Szecsi PB, Jørgensen M, Klajnbard A et al. Haemostatic reference intervals in pregnancy. Thromb Haemost 2010;103:718-27.

11 Armstrong S, Fernando R, Ashpole K, Simons R, Columb M. Assessment of coagulation in the obstetric population using ROTEM ${ }^{\circledR}$ thromboelastometry. Int J Obstet Anesth 2011;20:293-8.

12 Duarte RCF, Ferreira CN, Rios DRA, Reis HJD, Carvalho MDG. Thrombin generation assays for global evaluation of the hemostatic system: perspectives and limitations. Rev Bras Hematol Hemoter 2017:39:259-65.

13 Rizoli SB, Scarpelini S, Callum ] et al. Clotting factor deficiency in early trauma-associated coagulopathy. J Trauma 2011;71(5 Suppl 1):S427-34.

14 Haas T, Fries D, Tanaka KA et al. Usefulness of standard plasma coagulation tests in the management of perioperative coagulopathic bleeding: is there any evidence? $\mathrm{Br}$ ] Anaesth 2015;114:217-24.

15 Baksaas-Aasen K, Gall L, Eaglestone S et al. iTACTIC - implementing Treatment Algorithms for the Correction of Trauma-Induced Coagulopathy: study protocol for a multicentre, randomised controlled trial. Trials 2017;18:486.

16 Levi M, Opal SM. Coagulation abnormalities in critically ill patients. Crit Care 2006;10:222.

17 Levi M, Toh CH, Thachil J, Watson HG. Guidelines for the diagnosis and management of disseminated intravascular coagulation. British Committee for Standards in Haematology. Br J Haematol 2009;145:24-33.

18 Bick RL. Disseminated intravascular coagulation: objective clinical and laboratory diagnosis, treatment, and assessment of therapeutic response. Semin Thromb Hemost 1996;22:69-88.

19 Retter A, Barrett NA. The management of abnormal haemostasis in the ICU. Anaesthesia 2015;70(Suppl 1):121-7, e140-21.
20 Stanworth S], Walsh TS, Prescott RJ et al. Thrombocytopenia and platelet transfusion in UK critical care: a multicenter observational study. Transfusion 2013:53:1050-8.

21 Baharoglu MI, Cordonnier C, Al-Shahi Salman R et al. Platelet transfusion versus standard care after acute stroke due to spontaneous cerebral haemorrhage associated with antiplatelet therapy (PATCH): a randomised, open-label, phase 3 trial. Lancet 2016;387:2605-13.

22 Chakraverty R, Davidson S, Peggs K et al. The incidence and cause of coagulopathies in an intensive care population. $\mathrm{Br}$ J Haematol 1996;93:460-3.

23 Green L, Bolton-Maggs P, Beattie C et al. British Society of Haematology Guidelines on the spectrum of fresh frozen plasma and cryoprecipitate products: their handling and use in various patient groups in the absence of major bleeding. $\mathrm{Br}]$ Haematol 2018;181:54-67.

24 Stanworth SJ, Walsh TS, Prescott RJ et al. A national study of plasma use in critical care: clinical indications, dose and effect on prothrombin time. Crit Care 2011;15:R108.

25 Stanworth SJ, Desborough M, Somins G et al. Clinical bleeding and thrombin generation in admissions to critical care with prolonged prothrombin time: an exploratory study. Transfusion 2018, in press (doi: 10.1111/trf.14605).

26 De Pietri L, Bianchini M, Montalti R et al. Thrombelastographyguided blood product use before invasive procedures in cirrhosis with severe coagulopathy: A randomized, controlled trial. Hepatology 2016;63:566-73.

Address for correspondence: Dr Simon Stanworth, NHS Blood and Transplant, John Radcliffe Hospital, Headley Way, Headington, Oxford OX3 9BQ, UK.

Email: simon.stanworth@nhsbt.nhs.uk 